\title{
What is the Diagnostic Accuracy of Aspirations Performed on Hips With Antibiotic Cement Spacers?
}

\author{
Jared M. Newman MD, Jaiben George MBBS, Alison K. Klika MS, Stephen F. Hatem MD, \\ Wael K. Barsoum MD, W. Trevor North MD, Carlos A. Higuera MD
}

Received: 16 May 2016/Accepted: 14 September 2016/Published online: 26 September 2016

(C) The Association of Bone and Joint Surgeons \& 2016

\begin{abstract}
Background Periprosthetic joint infection is a serious complication after THA and commonly is treated with a two-stage revision. Antibiotic-eluting cement spacers are placed for local delivery of antibiotics. Aspirations may be performed before the second-stage reimplantation for identification of persistent infection. However, limited data exist regarding the diagnostic parameters of synovial fluid aspiration with or without saline lavage from a hip with an antibiotic-loaded cement spacer.

Questions/purposes We asked: (1) For hips with antibiotic cement spacers, does saline lavage influence the diagnostic validity of aspirations? (2) What is the diagnostic accuracy of preoperative aspirations performed on
\end{abstract}

One of the authors certifies that he (WKB), or a member of his immediate family, has or may receive payments or benefits, during the study period an amount of USD 100,001 to USD 1,000,000 from Stryker (Kalamazoo, MI, USA); an amount of USD 100,001 to USD 1,000,000 from Exactech (Gainesville, FL, USA); an amount of less than USD 10,000 from Zimmer (Warsaw, IN, USA); an amount of less than USD 10,000 from Custom Orthopaedic Solutions (Cleveland, OH, USA; an amount of USD 100,001 to USD 1,000,000 from KEF Healthcare (Westminster, CO, USA); amount of less than USD 10,000 from iVHR (Cleveland, OH, USA); an amount of less than USD 10,000 from PeerWell (San Francisco, CA, USA), and has received research support from Zimmer (Warsaw, IN, USA), DJO (Vista, CA, USA), and Orthosensor (Dania Beach, FL, USA). One of the authors (CAH) or a member of his immediate family, has or may receive payments or benefits, during the study period, an amount of less than USD 10,000 from KCI (San Antonio, TX, USA); an amount of less than USD 10,000 from Zimmer Biomet (Warsaw, IN, USA); an amount of less than USD 10,000 from Covance (Princeton, NJ, USA); an amount of less than USD 10,000 from Pfizer (New York, NY, USA); and has received research support funding from Stryker (Kalamazoo, MI, USA), Myoscience (Fresno, CA, USA), CD Diagnostics (Claymont, DE, USA), KCI (San Antonio, TX, USA), OREF (Rosemont, IL, USA), and Pacira (Parsippany, NJ, USA). hips with antibiotic cement spacers using the Musculoskeletal Infection Society (MSIS) criteria, stratified by saline and nonlavage? (3) For hips with antibiotic spacers, what are the optimal thresholds for synovial fluid white blood cell (WBC) count and polymorphonuclear neutrophil (PMN) percentage for diagnosing infections?

Methods One hundred seventy-four hips (155 patients) with antibiotic-eluting cement spacers inserted between October 2012 and July 2015 were reviewed. Of these, 98 hips (80 patients) met the inclusion criteria and were included in the analysis (77 nonlavage, 21 saline lavage aspirations). Laboratory data from the aspiration and preoperative workup and intraoperative details were collected. Infection status of each hip procedure was determined

All ICMJE Conflict of Interest Forms for authors and Clinical Orthopaedics and Related Research ${ }^{\mathbb{R}}$ editors and board members are on file with the publication and can be viewed on request.

Each author certifies that his or her institution approved or waived approval for the human protocol for this investigation and that all investigations were conducted in conformity with ethical principles of research.

Electronic supplementary material The online version of this article (doi:10.1007/s11999-016-5093-8) contains supplementary material, which is available to authorized users.

J. M. Newman, J. George, A. K. Klika ( $₫)$, W. K. Barsoum, W. Trevor North, C. A. Higuera

Department of Orthopaedic Surgery, Cleveland Clinic, A41, 9500 Euclid Avenue, Cleveland, OH 44195, USA e-mail: klikaa@ccf.org

\section{S. F. Hatem}

Department of Radiology, Cleveland Clinic, Cleveland, OH, USA 
based on a modified MSIS criteria using serologic, histologic, and intraoperative findings (sinus tract communicating with the joint at surgery or two positive intraoperative periprosthetic cultures with the same organism or two of the three following criteria: elevated erythrocyte sedimentation rate [ESR] [>30 mm/hour] and C-reactive protein $[\mathrm{CRP}][>10 \mathrm{mg} / \mathrm{L}]$, a single positive intraoperative periprosthetic tissue culture, or a positive histologic analysis of periprosthetic tissue [ $>5$ neutrophils per high power field]). The diagnostic parameters were calculated for the MSIS criteria thresholds for synovial fluid (ie, WBC count $>3000$ cells/ $\mu \mathrm{L}$ and PMN percentage $>80 \%$ ). Optimal thresholds were calculated for the corrected synovial WBC count and PMN percentage with a receiver operating characteristic curve. Separate analyses were performed for the hips with successful aspirations (nonlavage group) and those with saline lavage aspirations. Results The WBC count and PMN percentage were higher in hips with infection than in hips without infection when nonlavage aspirations were done (WBC count, 6680 cells $/ \mu \mathrm{L} \pm 6980$ cells $/ \mu \mathrm{L}$ vs $2001 \pm 4825$; mean difference, 4679; 95\% CI, 923-8436; $\mathrm{p}=0.015 ; \mathrm{PMN}$ percentage, $83 \% \pm 13 \%$ vs $44 \% \pm 30 \%$; mean difference, 39\%; 95\% CI, 39\%-49\%; p < 0.001) and the findings between infected and noninfected aspirations were not different when saline lavage aspirations were done (WBC count, 782 cells $/ \mu \mathrm{L} \pm 696$ vs 307 cells $/ \mu \mathrm{L} \pm 343$; mean difference, $475 ; 95 \% \mathrm{CI},-253$ to $1203 ; \mathrm{p}=0.161$; PMN percentage, $67 \% \pm 15 \%$ vs $58 \% \pm 28 \%$; mean difference, $10 \%$; $95 \% \mathrm{CI},-11 \%$ to $30 \%$; $=0.331)$. Aspirations performed without lavage yielded good diagnostic accuracy in all parameters (WBC count, 78\% [95\% CI, 70\%86\%]; PMN percentage. 79\% [95\% CI, 70\%-88\%]; positive culture: $84 \%$ [95\% CI, $81 \%-90 \%$ ]; at least one of the above: $79 \%$ [95\% CI, 70\%-88\%]); but in the saline lavage group, none had WBC counts above the threshold (diagnostic accuracies for WBC count, 0\%; PMN percentage, $71 \%$ [95\% CI, $62 \%-86 \%$ ]; positive culture, $76 \%$ [95\% CI, 76\%-86\%]; at least one: $71 \%$ [95\% CI, 57\%-91\%]). Because saline lavage did not result in differences between aspirations from infected and noninfected hips, we calculated the optimal thresholds in the nonlavage group only; the optimal threshold for synovial WBC count was 1166 cells $/ \mu \mathrm{L}$ and for synovial PMN the percentage was $68 \%$, which corresponds to WBC count diagnostic accuracy of $78 \%$ (95\% CI, 69\%-87\%) and PMN percentage accuracy of $78 \%$ (95\% CI, $69 \%-87 \%$ ).

Conclusions Because the MSIS criteria thresholds resulted in suboptimal sensitivities owing to a higher number of false negatives, we recommend considering lower WBC count and PMN percentage thresholds for hip-spacer aspirations. Furthermore, the WBC count and PMN percentage results from aspirations performed with saline lavage are not reliable for treatment decisions.

Level of Evidence Level III, diagnostic study.

\section{Introduction}

Hip aspirations for synovial fluid cell count and culture along with measurement of serum C-reactive protein (CRP) and erythrocyte sedimentation rate (ESR) typically are performed as screening tests for infection before revision THA [4, 8]. These aspirations have reported sensitivities ranging from $55 \%$ to $99 \%[3,5,9,13,25]$. The Musculoskeletal Infection Society (MSIS) criteria [20] is the most agreed upon and widely used standard for diagnosing periprosthetic joint infections and is based on the presence of one of two major criteria (ie, two positive periprosthetic cultures of the same organism with same phenotype and/or a sinus tract that communicates with the joint) or three of five minor criteria (ie, ESR and CRP, elevated synovial fluid white blood cell [WBC] count or positive $(++)$ change on leukocyte esterase strip, elevated synovial fluid polymorphonuclear neutrophil [PMN] percentage, a positive periprosthetic tissue histologic analysis, or one positive culture). Once infection is diagnosed, the current treatment algorithm includes two-stage revision, where the implants are removed, and antibiotic-loaded cement spacers are placed for local delivery of antibiotics to infected tissue [7, 17, 24].

However, possible persistent antibiotic elution before the second stage may yield false negative preoperative hip aspiration results and lead to an increased risk of postreimplantation persistent infection. The MSIS guidelines' thresholds have not been validated in the diagnosis of persistent infection after stage-one revisions in THA where antibiotic cement spacers are used. To aid in diagnosis, aspirations also may be done before the second-stage reimplantation to determine if there is a persistent infection $[16,18,26]$. Despite the assistance of fluoroscopic guidance, hip aspiration can be complicated by a lack of accessible synovial fluid or a "dry tap" [4]. As a solution to this problem, it has been suggested that sterile saline can be injected in these "dry taps" and then reaspirated [1, 6, 31], although this is controversial owing to the fear of inaccurate sampling and contamination $[1,28]$. To the best of our knowledge, diagnostic parameters of synovial fluid aspiration with and without saline lavage from a hip with an antibiotic-loaded cement spacer have not been described.

Therefore, we asked: (1) For hips with antibiotic cement spacers, does saline lavage influence the diagnostic validity of aspirations? (2) What is the diagnostic accuracy of preoperative aspirations performed on hips with antibiotic 
cement spacers using the MSIS criteria, stratified by saline and no lavage? (3) For hips with antibiotic spacers, what are the optimal thresholds for WBC count and percent PMNs for diagnosing infections?

\section{Methods}

\section{Study Design and Setting}

Once institutional review board approval was obtained, we retrospectively identified 174 hips (155 patients) with antibiotic-eluting cement spacers that were placed by one of 14 surgeons who perform revision joint replacement surgery, between October 2012 and July 2015, at a large academic hospital. During the first-stage surgery for the cement spacer, there are typically three to four bags of antibiotic cement used with $3.6 \mathrm{~g}$ of tobramycin and 2 to 3 $\mathrm{g}$ of vancomycin per bag of cement; in cases of fungi, 600 $\mathrm{mg}$ of amphotericin B with either $3.6 \mathrm{~g}$ of tobramycin or 3 $\mathrm{g}$ of vancomycin per bag of cement is used. Postoperatively, patients typically received intravenous antibiotics for 6 to 8 weeks with an antibiotic holiday 2 weeks before aspiration. The average time between the stages was 125.6 days (range, 49-503 days); this wide range was attributable to some of the patients' comorbidities that prevented the second-stage surgery from being performed earlier.

\section{Participants and Study Subjects}

Patients were sequentially excluded from the study if they did not have a second-stage surgery (reimplantation or spacer exchange) after the aspiration $(\mathrm{n}=19)$; did not have an aspiration performed $(\mathrm{n}=22)$; no aspiration was obtained, even with saline lavage $(n=2)$; no synovial fluid WBC count or synovial PMN percentage owing to insufficient fluid aspirated $(\mathrm{n}=32)$; and a successful aspiration was performed after which a saline lavage aspiration also was performed but the results did not specify which aspiration sample was used $(n=1)$. The decision to aspirate was not simply surgeon dependent, there were patient factors that influenced this decision. Therefore, nearly every surgeon included in the study had some cases in which he or she chose not to obtain a preoperative aspiration.

\section{Variables, Outcome Measures, Data Sources, and Bias}

A thorough chart review was conducted to collect key laboratory data at the time of aspiration and intraoperative details. These included: serum WBC and red blood cell (RBC) counts, ESR and CRP, synovial WBC and RBC counts, and the corrected synovial fluid WBC count as described by Ghanem et al. [12] was calculated (corrected $\mathrm{WBC}$ count $=\mathrm{WBC}$ synovial fluid $-[\mathrm{WBC}$ count blood $\times$ RBC synovial fluid/RBC blood]). Hereafter, all WBC counts will refer to the corrected WBC count. In addition, the synovial fluid culture regardless whether saline lavage was performed, and intraoperative details including the presence or absence of a sinus tract, histologic analysis of periprosthetic tissue, and culture results were collected. All hip-spacer arthroplasty aspirations were performed using fluoroscopic guidance. Saline lavage technique was used if the needle was confirmed to be positioned appropriately using fluoroscopy and no fluid could be aspirated; the saline was injected in the hip and reaspirated. The reaspirated fluid then was used in the aforementioned analysis. For the purposes of this study, a hip was considered infected based on a modified MSIS criteria: if there was one of the following major criteria including a sinus tract communicating with the joint at surgery or two positive intraoperative periprosthetic cultures with the same organism, or if there were two of the three of the following minor criteria: elevated ESR ( $>30 \mathrm{~mm} /$ hour) and CRP $(>$ $10 \mathrm{mg} / \mathrm{L}$ ), a single positive intraoperative periprosthetic tissue culture, or a positive histologic analysis of periprosthetic tissue ( $>5$ neutrophils per high power field) [20]. This was considered the reference standard for evaluating the diagnostic accuracy of synovial fluid parameters (WBC count, PMN percentage, and culture) obtained from the aspiration.

\section{Statistical Analysis, Study Size}

We first compared infected and not infected hips in terms of the WBC count and PMN percentage in aspirations performed with nonlavage and saline lavage. Then, sensitivity, specificity, positive predictive value (PPV), negative predictive value (NPV), and accuracy of synovial fluid WBC count and PMN percentage were calculated using the thresholds specified according to the MSIS criteria [20]. Additionally, for hips with antibiotic spacers, to determine the optimal synovial fluid WBC and PMN percentage thresholds, a receiver operating characteristic (ROC) curve was used. The optimal thresholds were defined as the cutoff point that provided the highest Youden's J statistic, which determined the maximum sensitivity and specificity of the test (Fig. 1). The 95\% CIs for the thresholds and diagnostic parameters were computed with 2000 stratified bootstrap replicates. The diagnostic parameters at the optimal threshold were compared with those at the MSIS threshold using the McNemar test (sensitivity, specificity, and 


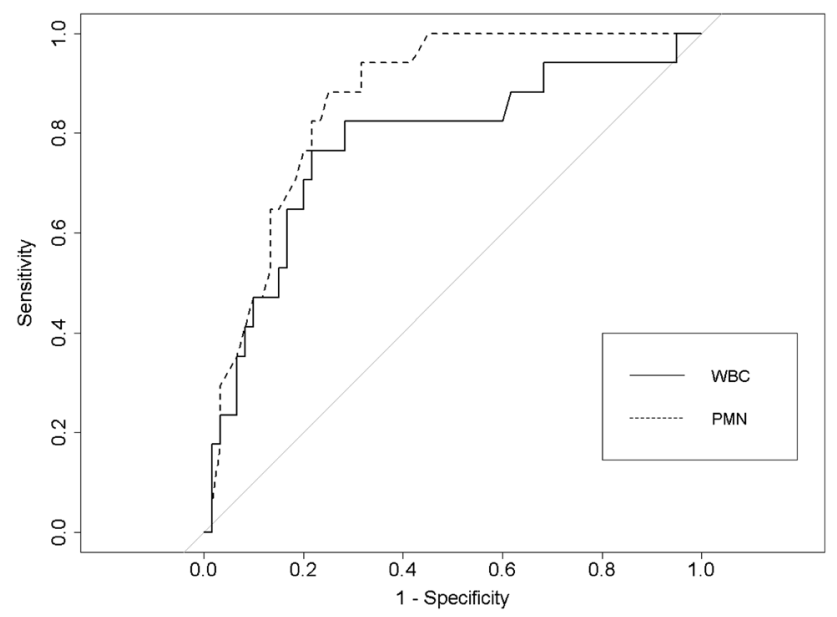

Fig. 1 A receiver operating characteristic curve was used to determine the optimal synovial fluid WBC count (solid line) and PMN percentage (dashed line) thresholds for the nonlavage group. $\mathrm{WBC}=$ white blood cell; PMN = polymorphonuclear leukocytes.

accuracy) or generalized score statistic (PPV and NPV). All analyses were performed using the ' $p$ ROC' package in the R software (version 3.1.3; R Foundation for Statistical Computing, Vienna, Austria) [23]. A p value less than 0.05 was used to determine statistical significance.

\section{Demographics, Description of Study Population}

A total of 98 hips (80 patients) were included in the study; the aspirations were considered as different events in the 80 patients and therefore, the number of hips, not the number of patients, was considered the unit of study. Successful aspirations were performed on 77 hips (78.6\%; nonlavage group) and saline lavage was performed in 21 hips $(21.4 \%)$. The mean age of patients at the time of aspiration was 64.2 years $( \pm 12.5$ years) and the cohort included 58 (59\%) males and $40(41 \%)$ females. There were 57 right and 41 left hips. Twenty-three of the 98 hips (23.5\%) had persistent infection, as per our modified MSIS criteria; nine hips were diagnosed as infected with only the minor criteria (ie, at least two of three minor criteria, as per above) with the most common minor criteria being positive histologic analysis of periprosthetic tissue in 15 hips and elevated ESR and CRP in 16 hips. Intraoperative cultures were positive in 24 hips (24.5\%), including the six hips with positive aspiration cultures. The remaining 74 hips had negative cultures from aspiration and intraoperative samples. The organisms isolated from the synovial aspiration and intraoperative samples were recorded. (Appendix 1. Supplemental material is available with the online version of $C O R R^{\circledR}$ ). Of the persistently infected 23 hips, 16 underwent spacer exchange, one had spacer removal and Girdlestone procedure, one had a disarticulation, and five underwent reimplantation at the secondstage surgery; this was attributable to either improvement in inflammatory markers and the surgeon thought that reimplantation would be the best option, the patient would not be able to handle another surgery, or results of the intraoperative cultures were not received until after the surgery and the criteria for infection was met postoperatively. There were 65 hips that had no persistent infection as per the modified MSIS criteria we used, and of those, seven underwent additional surgery for wound issues and/ or infection. Ten hips were considered to be without persistent infection based on the modified MSIS criteria, but had spacer exchange; four hips were considered infected based on the MSIS criteria. The other six hips did not meet our criteria, but based on laboratory results and intraoperative findings, the surgeons decided to perform a spacer exchange; one hip had purulence in the joint, three had positive frozen sections intraoperatively, one had an elevated ESR and CRP, and one had a positive culture and elevated ESR.

\section{Results}

When saline lavage aspirations were performed, the findings between infected and noninfected hips were not significantly different (WBC count, 782 cells $/ \mu \mathrm{L} \pm 696$ vs 307 cells $/ \mu \mathrm{L} \pm 343$; mean difference, $475 ; 95 \% \mathrm{CI},-253$ to $1203 ; \mathrm{p}=0.161 ;$ PMN percentage, $67 \% \pm 15 \%$ vs $58 \%$ $\pm 28 \%$; mean difference, $10 \%$; $95 \% \mathrm{CI},-11 \%$ to $30 \%$; $\mathrm{p}=$ 0.331). In the saline lavage group, six of 21 (29\%) hips had persistent infection, and in the nonlavage group 17 of 77 (22\%) had persistent infection. The WBC count and PMN percentage were higher in the hips with infection than in hips without infection when nonlavage aspirations were done (WBC count, 6680 cells $/ \mu \mathrm{L} \pm 6980$ cells $/ \mu \mathrm{L}$ vs 2001 \pm 4825; mean difference, 4679; 95\% CI, 923-8436; $\mathrm{p}=$ 0.015 ; PMN percentage, $83 \% \pm 13 \%$ vs $44 \% \pm 30 \%$; mean difference, 39\%; 95\% CI, 39\%-49\%; p < 0.001) (Table 1). In the infected group, saline lavage produced lower synovial WBC counts compared with nonlavage aspirations (saline lavage, 782 cells $/ \mu \mathrm{L} \pm 696$; nonlavage, 6680 cells $/ \mu \mathrm{L} \pm 6980$; mean difference, $5897 ; 95 \% \mathrm{CI}$, 2274-9521; $\mathrm{p}=0.003$ ), but there was no difference in PMN percentage (saline lavage, $67 \% \pm 15 \%$; nonlavage, $83 \% \pm 13 \%$; mean difference, $16 \%$; $95 \%$ CI, $0 \%-32 \%$; $=$ $0.053)$.

Using thresholds from the MSIS criteria, nonlavage aspirations yielded good diagnostic accuracy in all parameters (WBC count, 78\% [95\% CI, 70\%-86\%]; PMN percentage, $79 \%$ [95\% CI, 70\%-88\%]; positive culture, $84 \%$ [ $95 \%$ CI, $81 \%-90 \%$ ]; at least one of the above, $79 \%$ 
Table 1. Synovial fluid parameters in saline lavage and nonlavage aspirations based on infection status

\begin{tabular}{|c|c|c|c|c|}
\hline Variable & Infected ( $\mathrm{n}=23$ hips) & Not infected $(\mathrm{n}=75$ hips $)$ & Mean difference $(95 \% \mathrm{CI})$ & $\mathrm{p}$ Value \\
\hline \multicolumn{5}{|c|}{ WBC count (cells/ $\mu \mathrm{L})$} \\
\hline Saline lavage & $782 \pm 696(n=6)$ & $307 \pm 343(\mathrm{n}=15)$ & $475(-253$ to 1203$)$ & 0.161 \\
\hline Nonlavage & $6680 \pm 6980(n=17)$ & $2001 \pm 4825(n=60)$ & $4679(923-8436)$ & 0.015 \\
\hline \multicolumn{5}{|c|}{ PMN percentage $(\%)$} \\
\hline Saline lavage & $67 \pm 15(\mathrm{n}=6)$ & $58 \pm 28(\mathrm{n}=15)$ & $10(-11$ to 30$)$ & 0.331 \\
\hline Nonlavage & $83 \pm 13(\mathrm{n}=17)$ & $44 \pm 30(n=60)$ & $39(39-49)$ & $<0.001$ \\
\hline
\end{tabular}

Values = mean $\pm \mathrm{SD} ; \mathrm{WBC}=$ white blood cell; $\mathrm{PMN}=$ polymorphonuclear cells.

Table 2. Diagnostic accuracy of synovial fluid using the MSIS criteria

\begin{tabular}{|c|c|c|c|c|}
\hline Parameter & WBC count $>3000$ cells $/ \mu \mathrm{L}$ & PMN percentage $>80 \%$ & Positive culture & At least one* \\
\hline \multicolumn{5}{|l|}{ Nonlavage group ( $\mathrm{n}=77$ hips) } \\
\hline Number of hips (\%) & $16(21 \%)$ & $25(32 \%)$ & $5(6.5 \%)$ & $27(35 \%)$ \\
\hline Sensitivity & $47 \%(24-70)$ & $76 \%(53-94)$ & $30 \%(12-53)$ & $82 \%(65-100)$ \\
\hline Specificity & $87 \%(78-95)$ & $80 \%(70-90)$ & $100 \%(100-100)$ & $78 \%(64-87)$ \\
\hline Positive predictive value & $50 \%(31-73)$ & $52 \%(38-68)$ & $100 \%(100-100)$ & $52 \%(40-67)$ \\
\hline Negative predictive value & $85 \%(80-91)$ & $92 \%(86-98)$ & $83 \%(80-88)$ & $94 \%(88-100)$ \\
\hline Accuracy & $78 \%(70-86)$ & $79 \%(70-88)$ & $84 \%(81-90)$ & $79 \%(70-88)$ \\
\hline Area under the curve & $0.668(0.54-0.80)$ & $0.782(0.67-0.90)$ & $0.647(0.54-0.76)$ & $0.803(0.70-0.91)$ \\
\hline \multicolumn{5}{|c|}{ Saline lavage group ( $\mathrm{n}=21$ hips) } \\
\hline Number of hips (\%) & $0(0 \%)$ & $5(24 \%)$ & $1(4.8 \%)$ & $6(29 \%)$ \\
\hline Sensitivity & N/A & $33 \%(17-67)$ & $17 \%(17-50)$ & $50 \%(17-83)$ \\
\hline Specificity & N/A & $87 \%(60-100)$ & $100 \%(100-100)$ & $80 \%(60-100)$ \\
\hline Positive predictive value & N/A & $50 \%(33-100)$ & $100 \%(100-100)$ & $50 \%(33-100)$ \\
\hline Negative predictive value & N/A & $78 \%(72-88)$ & $75 \%(75-83)$ & $81 \%(73-93)$ \\
\hline Accuracy & N/A & $71 \%(62-86)$ & $76 \%(76-86)$ & $71 \%(57-91)$ \\
\hline Area under the curve & N/A & $0.567(0.335-0.798)$ & $0.583(0.42-0.747)$ & $0.650(0.407-0.893)$ \\
\hline
\end{tabular}

Thresholds and 95\% CIs are shown in parentheses; MSIS = Musculoskeletal Infection Society; WBC $=$ white blood cell; PMN = polymorphonuclear leukocytes; N/A = not applicable. *Having at least one of the following: WBC count $>3000$ cells $/ \mu \mathrm{L}, \mathrm{PMN}$ percentage $>80 \%$, or positive synovial fluid culture.

[95\% CI, 70\%-88\%]); but in the saline lavage group, none had WBC counts above the threshold of 3000 cells $/ \mu \mathrm{L}$ (diagnostic accuracies for WBC count, $0 \%$; PMN \%, $71 \%$ [95\% CI, 62\%-86\%]; positive culture, 76\% [95\% CI, 76\%$86 \%$ ]; at least one, $71 \%$ [95\% CI, 57\%-91\%]) (Table 2).

In the nonlavage group, the optimal threshold for synovial fluid WBC count was 1166 cells $/ \mu \mathrm{L}$ and for synovial PMN percentage was $68 \%$ (Table 3 ), which corresponds to a WBC count diagnostic accuracy of $78 \%$ (95\% CI, 69\%$87 \%$ ) and PMN percentage accuracy of $78 \%$ (95\% CI, 69\%-87\%). The calculations for the optimal thresholds in the saline lavage group were not performed because the initial analysis failed to reveal any differences between the infected and noninfected hips when aspirated using saline lavage. The diagnostic parameters using the optimal thresholds that were calculated in the nonlavage group were compared with the MSIS criteria thresholds
Table 3. Diagnostic parameters of synovial fluid WBC count and PMN percentage at optimal thresholds

\begin{tabular}{lll}
\hline Parameter & WBC count & PMN percentage \\
\hline Optimal threshold & 1166 cells $/ \mu \mathrm{L}$ & $68 \%$ \\
Sensitivity & $76 \%(53-94)$ & $88 \%(71-100)$ \\
Specificity & $78 \%(67-88)$ & $75 \%(63-85)$ \\
Positive predictive value & $50 \%(37-65)$ & $50 \%(40-64)$ \\
Negative predictive value & $92 \%(85-98)$ & $96 \%(90-100)$ \\
Accuracy & $78 \%(69-87)$ & $78 \%(69-87)$ \\
Area under the curve & $0.774(0.69-0.87)$ & $0.852(0.72-0.91)$
\end{tabular}

$\mathrm{WBC}=$ white blood cell; $\mathrm{PMNs}=$ polymorphonuclear leukocytes; 95\% CIs are shown in parentheses.

(Table 4). When compared with the MSIS criteria thresholds, our new recommended threshold for synovial fluid WBC count had better sensitivity (76\% [95\% CI, 53\%- 
Table 4. Comparison of sensitivity and specificity using the MSIS threshold and the new threshold

\begin{tabular}{|c|c|c|c|}
\hline Parameter & MSIS threshold & New threshold & $\mathrm{p}$ Value \\
\hline \multicolumn{4}{|l|}{ WBC count } \\
\hline Threshold & 3000 cells $/ \mu \mathrm{L}$ & 1166 cells $/ \mu \mathrm{L}$ & N/A \\
\hline Sensitivity & $47 \%(24-70)$ & $76 \%(53-94)$ & 0.025 \\
\hline Specificity & $87 \%(78-95)$ & $78 \%(67-88)$ & 0.025 \\
\hline Positive predictive value & $50 \%(31-73)$ & $50 \%(37-65)$ & 1 \\
\hline Negative predictive value & $85 \%(80-91)$ & $92 \%(85-98)$ & 0.037 \\
\hline Accuracy & $78 \%(70-86)$ & $78 \%(69-87)$ & 0.739 \\
\hline \multicolumn{4}{|l|}{ PMN percentage } \\
\hline Threshold & $80 \%$ & $68 \%$ & N/A \\
\hline Sensitivity & $76 \%(53-94)$ & $88 \%(71-100)$ & 0.157 \\
\hline Specificity & $80 \%(70-90)$ & $75 \%(63-85)$ & 0.083 \\
\hline Positive predictive value & $52 \%(38-68)$ & $50 \%(40-64)$ & 0.625 \\
\hline Negative predictive value & $92 \%(86-98)$ & $96 \%(90-100)$ & 0.183 \\
\hline Accuracy & $79 \%(70-88)$ & $78 \%(69-87)$ & 0.655 \\
\hline
\end{tabular}

MSIS = Musculoskeletal Infection Society; $\mathrm{WBC}=$ white blood cell; PMN = polymorphonuclear leukocytes; N/A= not applicable; 95\% CIs presented in parentheses.

94\%] vs $47 \%$ [95\% CI, 24\%-70\%], p = 0.025), lower specificity (78\% [95\% CI, 67\%-88\%] vs $87 \%$ [95\% CI, 78\%-95\%], $\mathrm{p}=0.025)$, but without sacrificing diagnostic accuracy $(78 \%$ [95\% CI, 69\%-87\%] vs $78 \%$ [95\% CI, $70 \%-86 \%], \mathrm{p}=0.739)$. However, there was no difference in diagnostic accuracy in PMN percentage when using our new threshold (new threshold vs MSIS, sensitivity: $88 \%$ [95\% CI, 71\%-100\%] vs 76\% [95\% CI, 53\%-94\%], p = 0.157; specificity: $75 \%$ [95\% CI, $63 \%-85 \%$ ] vs $80 \%$ [ $95 \%$ CI, 70\%-90\%], $\mathrm{p}=0.083$; accuracy: $78 \%$ [95\% CI, 69\%$87 \%$ ] vs $79 \%$ [ $95 \% \mathrm{CI}, 70 \%-88 \%$ ], $\mathrm{p}=0.655$ ).

\section{Discussion}

The recognition of persistent infection after hip-spacer arthroplasty and appropriate antibiotic treatment is important for successful reimplantation. Although the MSIS criteria are considered the best standard we have for diagnosing acute and chronic periprosthetic joint infections in total joint arthroplasties, it was not designed to address joints with antibiotic-eluting spacers. There are limited studies that have evaluated methods to determine whether the periprosthetic joint infection has been effectively treated before the second-stage surgery $[11,15,16,26]$. No previous studies, of which we are aware, have examined the utility of preoperative hip aspirations with or without saline lavage for diagnosing persistent infection in hips with antibiotic cement spacers, and no cutoff values for WBC count and PMN percentage have been established. Therefore, we aimed to determine the optimal threshold values for synovial fluid WBC count and PMN percentage in hips with antibiotic cement spacers during the interim period of a two-stage hip revision for periprosthetic joint infections.

There are notable limitations to this study. First, this was a retrospective study subject to selection bias. Although we analyzed all saline lavage aspirates as a single group, the amount of saline injected is operator dependent and may influence the synovial fluid analysis. In addition, although all of the saline lavage aspirates were sent for culture analysis, not all were sent for synovial fluid analysis because of insufficient fluid, therefore limiting the inclusion of those samples. Second, we did attempt to determine whether the tests performed differently between men and women. Third, the study was limited by the sample size because a periprosthetic joint infection is a relatively rare complication. Owing to the requirement for intraoperative samples for diagnosing infection, patients who did not undergo a second-stage surgery were not included, regardless of their aspiration results. Fourth, it would have been clinically useful to compare the sensitivities and specificities of the MSIS thresholds with the new thresholds in the hips that had saline lavage, but given that all WBC counts in the saline lavage group were less than 3000 cells $/ \mu \mathrm{L}$ this was not possible. Fifth, it is not the standard of care to perform an aspiration before the second-stage surgery; the decision to aspirate is not simply surgeon dependent. There are also patient factors which influence this decision. Therefore, nearly every surgeon included in the study had some cases in which he or she chose not to get a preoperative aspiration. Sixth, although we used a 
modified MSIS criteria to diagnose persistent infection before the second-stage surgery, it is possible that some of the hips deemed to be not infected by this modified MSIS criteria might have been infected. If we also considered patients who had a failure as cases with persistent infection when followed long-term, the diagnostic parameters in this study might have been different and the failure rates may have been higher. Because positive cultures and positive MSIS criteria at the time of the second-stage reimplantation have been reported to increase the risk of subsequent failure, the criteria we used can be considered to be a reliable indicator of persistent infection [10, 29]. Moreover, changing the diagnostic criteria to include long-term followup is unlikely to change the conclusions of our study that a lower threshold for synovial fluid parameters should be considered in hips with antibiotic cement spacers, as the same diagnostic criteria was used when comparing the MSIS thresholds with the new thresholds

Saline lavage may produce a dilutional effect on synovial fluid samples. Previous studies on hip aspirations have described using saline lavage injection and reaspiration when dry taps are encountered [1, 6, 19, 22, 27]. Although this saline lavage technique has been reported to provide accurate results [1], there may be discrepancies attributable to dilution of the aspiration sample that potentially might lead to false negative results $[1,22]$. Ali et al. [1] examined 73 patients who underwent hip aspiration and $32 \%$ of those who had saline lavage performed had a dry or inadequate initial aspiration. They compared the culture results with the intraoperative tissue cultures and reported a sensitivity, specificity, PPV, NPV, and accuracy of $83 \%, 82 \%, 63 \%, 93 \%$, and $83 \%$, respectively. No comparison was made with respect to WBC counts or PMN percentage. In the current study, the optimal thresholds for the saline lavage group for WBC count and PMN percentage were not calculated because there were no differences between the infected and noninfected hips with antibiotic spacers. Based on these results, we cannot recommend that WBC count and PMN percentages obtained from saline lavage in hips with antibiotic spacers be used in clinical decision making for identification of persistent periprosthetic joint infections.

In nonlavage hip-spacer aspirations, the MSIS criteria showed low sensitivities for the WBC count and PMN percentage, but with reasonable accuracies. Previous studies have examined the use of preoperative hip aspirations in helping to diagnose periprosthetic joint infections $[1,3,25,30,33]$. It has been suggested that preoperative aspirations should be performed on select patients with an intermediate to high risk of infection based on clinical and radiographic findings $[1,3,14]$. Moreover, in patients who have an antibiotic spacer in place for infection, preoperative aspiration before the second-stage procedure may help identify unresolved infections [16, 18]. Mont et al. [18] reported that cultures from preoperative aspirations before reimplantation reduced the rate of recurrent infection after the second-stage surgery by allowing clinicians to identify patients with persistent infections. Along with synovial fluid cultures, the synovial fluid WBC count and PMN percentage are useful tests for identifying persistent infections at the time of the second-stage surgery for infected THA [26]. Shukla et al. [26] reported on patients with hip spacers who had intraoperative aspirations during the second-stage surgery and found the WBC count sensitivity, specificity, and accuracy to be $78 \%, 96 \%$, and $94 \%$, respectively, and PMN percentage sensitivity, specificity, and accuracy of $78 \%, 82 \%$, and $82 \%$, respectively. The lower sensitivities reported in our study and by Shukla et al. [26] may be attributable to a high false negative rate owing to the antibiotic spacer elution, which can elute antibiotics for up to 8 weeks [2].

In nonlavage hip aspirations, a lower threshold for the WBC count gave higher sensitivity compared with the current MSIS-suggested thresholds. Prior studies have reported on the optimal thresholds for aspiration WBC count and PMN percentage in THA [21, 25, 32]. Schinsky et al. [25] investigated 201 painful THAs and performed intraoperative aspirations on all of the patients at the time of revision surgery. They found the optimal threshold for diagnosing infection was a WBC count of 3000 cells $/ \mu \mathrm{L}$. However, patients with an antibiotic spacer in place were excluded. Parvizi et al. [21] reported on aspirations performed on hips and knees with a high suspicion of being infected and found the cutoff for optimal accuracy for WBC count of 1760 cells $/ \mu \mathrm{L}$ and PMN percentage of $73 \%$ for diagnosing periprosthetic joint infections. Similarly, another study found the optimal threshold for diagnosing a periprosthetic joint infection to be a WBC count of 1700 cells $/ \mu \mathrm{L}$ and a PMN percentage of $65 \%$ [32]. However, there are few studies with results of aspirations performed before the second-stage surgery, while an antibiotic spacer is in place [11, 15, 26]. Shukla et al. [26] performed intraoperative aspirations before arthrotomy during the secondstage procedure and determined an optimal threshold for WBC count of 3528 cells $/ \mu \mathrm{L}$ and PMN percentage of $79 \%$. Kusuma et al. [15] reported on patients with knee periprosthetic joint infections who were treated with twostage revision surgery and had repeat aspirations performed on knees with antibiotic spacers, before the second surgery. They found the optimal thresholds for WBC count and PMN percentage to be 1102.5 cells $/ \mu \mathrm{L}$ and $71.5 \%$, respectively. We found results comparable to those reported by Kusuma et al. [15], with an optimal threshold for WBC count of 1166 cells $/ \mu \mathrm{L}$ and PMN percentage of $68 \%$.

The validity of the aspiration WBC count and PMN percentage results performed with saline lavage is not reliable 
and should not be relied on for decision-making purposes. We recommend considering lower WBC count and PMN percentage thresholds for hip-spacer aspirations, as the MSIS criteria thresholds resulted in a higher number of false negative results. Further prospective studies with larger cohorts may be required to determine the optimal thresholds to diagnose persistent infection before reimplantation surgery.

\section{References}

1. Ali F, Wilkinson JM, Cooper JR, Kerry RM, Hamer AJ, Norman $\mathrm{P}$, Stockley I. Accuracy of joint aspiration for the preoperative diagnosis of infection in total hip arthroplasty. J Arthroplasty. 2006;21:221-226.

2. Anagnostakos K, Kelm J. Enhancement of antibiotic elution from acrylic bone cement. J Biomed Mater Res B Appl Biomater. 2009;90:467-475.

3. Barrack RL, Jennings RW, Wolfe MW, Bertot AJ. The Coventry Award: the value of preoperative aspiration before total knee revision. Clin Orthop Relat Res. 1997;345:8-16.

4. Bauer TW, Parvizi J, Kobayashi N, Krebs V. Diagnosis of periprosthetic infection. J Bone Joint Surg Am. 2006;88:869-882.

5. Bernard L, Lübbeke A, Stern R, Bru JP, Feron JM, Peyramond D, Denormandie P, Arvieux C, Chirouze C, Perronne C, Hoffmeyer P; Groupe D'Etude Sur L'Ostéite. Value of preoperative investigations in diagnosing prosthetic joint infection: retrospective cohort study and literature review. Scand J Infect Dis. 2004;36:410-416.

6. Brandser EA, El-Khoury GY, FitzRandolph RL. Modified technique for fluid aspiration from the hip in patients with prosthetic hips. Radiology. 1997;204:580-582.

7. Buchholz HW, Elson RA, Heinert K. Antibiotic-loaded acrylic cement: current concepts. Clin Orthop Relat Res. 1984;190:96108.

8. Cipriano CA, Brown NM, Michael AM, Moric M, Sporer SM, Della Valle CJ. Serum and synovial fluid analysis for diagnosing chronic periprosthetic infection in patients with inflammatory arthritis. J Bone Joint Surg Am. 2012;94:594-600.

9. Della Valle CJ, Sporer SM, Jacobs JJ, Berger RA, Rosenberg AG, Paprosky WG. Preoperative testing for sepsis before revision total knee arthroplasty. J Arthroplasty. 2007;22(6 suppl 2):90-93.

10. George J, Kwiecien G, Klika AK, Ramanathan D, Bauer TW, Barsoum WK, Higuera CA. Are frozen sections and MSIS criteria reliable at the time of reimplantation of two-stage revision arthroplasty? Clin Orthop Relat Res. 2016;474:1619-1626.

11. Ghanem E, Azzam K, Seeley M, Joshi A, Parvizi J. Staged revision for knee arthroplasty infection: what is the role of serologic tests before reimplantation? Clin Orthop Relat Res. 2009;467:1699-1705.

12. Ghanem E, Houssock C, Pulido L, Han S, Jaberi FM, Parvizi J. Determining "true" leukocytosis in bloody joint aspiration. $J$ Arthroplasty. 2008;23:182-187.

13. Ghanem E, Parvizi J, Burnett RSJ, Sharkey PF, Keshavarzi N, Aggarwal A, Barrack RL. Cell count and differential of aspirated fluid in the diagnosis of infection at the site of total knee arthroplasty. J Bone Joint Surg Am. 2008;90:1637-1643.

14. Gould ES, Potter HG, Bober SE. Role of routine percutaneous hip aspirations prior to prosthesis revision. Skeletal Radiol. 1990;19:427-430.

15. Kusuma SK, Ward J, Jacofsky M, Sporer SM, Della Valle CJ. What is the role of serological testing between stages of two- stage reconstruction of the infected prosthetic knee? Clin Orthop Relat Res. 2011;469:1002-1008.

16. Lonner JH, Siliski JM, Della Valle C, DiCesare P, Lotke PA. Role of knee aspiration after resection of the infected total knee arthroplasty. Am J Orthop (Belle Mead NJ). 2001;30:305-309.

17. Masri BA, Duncan CP, Beauchamp CP. Long-term elution of antibiotics from bone-cement: an in vivo study using the prosthesis of antibiotic-loaded acrylic cement (PROSTALAC) system. J Arthroplasty. 1998;13:331-338.

18. Mont MA, Waldman BJ, Hungerford DS. Evaluation of preoperative cultures before second-stage reimplantation of a total knee prosthesis complicated by infection: a comparison-group study. $J$ Bone Joint Surg Am. 2000;82:1552-1557.

19. Mulcahy DM, Fenelon GC, McInerney DP. Aspiration arthrography of the hip joint: its uses and limitations in revision hip surgery. J Arthroplasty. 1996;11:64-68.

20. Parvizi J, Gehrke T; International Consensus Group on Periprosthetic Joint Infection. Definition of periprosthetic joint infection. J Arthroplasty. 2014;29:1331.

21. Parvizi J, Ghanem E, Menashe S, Barrack RL, Bauer TW. Periprosthetic infection: what are the diagnostic challenges? $J$ Bone Joint Surg Am. 2006;88(suppl 4):138-147.

22. Roberts P, Walters AJ, McMinn DJ. Diagnosing infection in hip replacements: the use of fine-needle aspiration and radiometric culture. J Bone Joint Surg Br. 1992;74:265-269.

23. Robin X, Turck N, Hainard A, Tiberti N, Lisacek F, Sanchez JC, Müller M. pROC: an open-source package for $\mathrm{R}$ and $\mathrm{S}+$ to analyze and compare ROC curves. BMC Bioinformatics. 2011;12:77.

24. Salvati EA, Callaghan JJ, Brause BD, Klein RF, Small RD. Reimplantation in infection: elution of gentamicin from cement and beads. Clin Orthop Relat Res. 1986;207:83-93.

25. Schinsky MF, Della Valle CJ, Sporer SM, Paprosky WG. Perioperative testing for joint infection in patients undergoing revision total hip arthroplasty. $J$ Bone Joint Surg Am. 2008;90:1869-1875.

26. Shukla SK, Ward JP, Jacofsky MC, Sporer SM, Paprosky WG, Della Valle CJ. Perioperative testing for persistent sepsis following resection arthroplasty of the hip for periprosthetic infection. J Arthroplasty. 2010;25(6 suppl):87-91.

27. Spangehl MJ, Masri BA, O'Connell JX, Duncan CP. Prospective analysis of preoperative and intraoperative investigations for the diagnosis of infection at the sites of two hundred and two revision total hip arthroplasties. J Bone Joint Surg Am. 1999;81:672-683.

28. Squire MW, Della Valle CJ, Parvizi J. Preoperative diagnosis of periprosthetic joint infection: role of aspiration. AJRAm $J$ Roentgenol. 2011;196:875-879.

29. Tan TL, Gomez MM, Manrique J, Parvizi J, Chen AF. Positive culture during reimplantation increases the risk of subsequent failure in two-stage exchange arthroplasty. J Bone Joint Surg Am. 2016;98:1313-1319.

30. Taylor T, Beggs I. Fine needle aspiration in infected hip replacements. Clin Radiol. 1995;50:149-152.

31. Tigges S, Stiles RG, Meli RJ, Roberson JR. Hip aspiration: a cost-effective and accurate method of evaluating the potentially infected hip prosthesis. Radiology. 1993;189:485-488.

32. Trampuz A, Hanssen AD, Osmon DR, Mandrekar J, Steckelberg JM, Patel R. Synovial fluid leukocyte count and differential for the diagnosis of prosthetic knee infection. Am J Med. 2004; 117:556-562.

33. Yi PH, Cross MB, Moric M, Levine BR, Sporer SM, Paprosky WG, Jacobs JJ, Della Valle CJ. Do serologic and synovial tests help diagnose infection in revision hip arthroplasty with metalon-metal bearings or corrosion? Clin Orthop Relat Res. 2015;473:498-505. 